\title{
BMJ Open The prognostic value of cardiac dysfunction assessed by bedside echocardiography in critically ill patients with COPD requiring mechanical ventilation: a study protocol
}

\author{
Zhongheng Zhang, Lin Chen, Kun Chen, Hongying Ni
}

To cite: Zhang Z, Chen L, Chen $\mathrm{K}$, et al. The prognostic value of cardiac dysfunction assessed by bedside echocardiography in critically ill patients with COPD requiring mechanical ventilation: a study protocol. BMJ Open 2014;4: 005359 . doi:10.1136/bmjopen-2014005359

- Prepublication history for this paper is available online. To view these files please visit the journal online (http://dx.doi.org/10.1136/ bmjopen-2014-005359)

Received 30 March 2014 Revised 8 August 2014 Accepted 20 August 2014

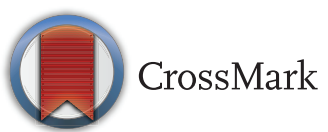

Department of Critical Care Medicine, Jinhua Municipal Central Hospital, Jinhua Hospital of Zhejiang University, Jinhua, Zhejiang, People's Republic of China

Correspondence to Dr Zhongheng Zhang; zh_zhang1984@hotmail.com

\section{ABSTRACT}

Introduction: Chronic obstructive lung disease is not only a major cause of morbidity and mortality, but is also the major reason for intensive care unit (ICU) admission. Cardiac function is often impaired in this disease, but its association with clinical outcome has not been fully established.

Methods and analysis: This is a prospective observational study conducted in a 47-bed mixed ICU of a tertiary academic teaching hospital. The study will be performed from January 2014 to December 2015. All patients meeting the diagnostic criteria of acute exacerbation of chronic obstructive pulmonary disease and admitted to the ICU are potentially eligible for the present study. The relevant demographics and laboratory measurements have been obtained. Transthoracic echocardiography was performed immediately after ICU admission by experienced intensivists. The Cox proportional hazard regression model has been fitted by using a stepwise forward selection and backward elimination technique. If linear assumption is not satisfied, the linear spline function will be used.

Ethics and dissemination: The study protocol was approved by the ethics committee of Jinhua municipal central hospital. The results will be published in a peer-reviewed journal and shared with the worldwide medical community.

Trial registration number: The study protocol is registered at ClinicalTrials.gov (NCT02099279).

\section{INTRODUCTION}

Chronic obstructive pulmonary disease (COPD) is a major cause of morbidity and mortality worldwide, with prevalence among the total population ranging from $7.8 \%$ to $19.7 \% .^{12}$ COPD is one of the most important causes of death in many countries. The Global Burden of Disease Study proposed that COPD, which was the sixth leading cause of death in 1990, will become the third leading cause of death by $2020 .^{3} 4$ Acute exacerbation of COPD (AECOPD) is an emergency that requires rapid medical intervention, and a significant proportion of patients with AECOPD require tracheal intubation and admission to the intensive care unit (ICU). Both mechanical ventilation and ICU admission are harbingers of adverse clinical outcome and are associated with a significant increase in financial cost. Factors such as male sex, age, low body mass index, chronic renal failure and lower limb oedema have been found to be the determinants of short-term mortality, whereas factors including diabetes mellitus, ischaemic heart disease, forced expiratory volume at $1 \mathrm{~s}$ and arterial oxygen partial pressure are predictors of long-term mortality. ${ }^{5}$

Cardiac dysfunction is commonly seen in mechanically ventilated patients with $\mathrm{AECOPD},{ }^{6}$ and it is associated with poor clinical outcomes such as weaning failure and high mortality rate. ${ }^{7}$ Several studies have proposed that cardiac evaluation should be incorporated into management strategy in patients undergoing mechanical ventilation because of the close interaction between the heart and lung. ${ }^{8-10}$ More recently, bedside echocardiography performed by intensivists has become increasingly popular in the management of critically ill patients, and its use has expanded our understanding of cardiac performance during mechanical ventilation. ${ }^{11} 12$

The present study aims to investigate the role of transthoracic echocardiography (TTE) measured cardiac indices in predicting clinical outcomes in patients with AECOPD requiring mechanical ventilation. Clinical outcomes include length of stay in the ICU and hospital, duration of mechanical ventilation and mortality. 


\section{STUDY DESIGN}

This is a prospective observational study conducted in a 47-bed mixed ICU of a tertiary academic teaching hospital. The hospital is the largest one in Jinhua city with over 2000 beds, providing tertiary medical care for a population of over five million. Our ICU enrolled medical and surgical patients with an expected annual admission of 1500 , and the proportion of patients admitted because of AECOPD accounted for around $17 \% .^{13}$ The study starts from January 2014, and is planned to last for a 2-year-period. All patients will be consecutively enrolled and followed up for the hospital stay.

\section{STUDY POPULATION}

All patients meeting the diagnostic criteria of AECOPD and admitted to the ICU are potentially eligible for the present study. The diagnosis of AECOPD is based on that defined in the Global Initiative for Chronic Obstructive Pulmonary Disease (GOLD COPD guideline, updated in 2014: From the Global Strategy for the Diagnosis, Management and Prevention of COPD, Global Initiative for Chronic Obstructive Lung Disease (GOLD) 2014. Available from: http://www.goldcopd.org/). COPD is "characterised by persistent airflow limitation that is usually progressive and associated with an enhanced chronic inflammatory response in the airways and the lung to noxious particles or gases.' AECOPD is defined using definitive criteria with at least two of the following major symptoms: increased dyspnoea, increased sputum purulence, increased sputum volume; or one major and one minor symptom: nasal discharge/congestion, wheeze, sore throat and cough for at least two consecutive days. ${ }^{14}$

In our institution, patients with COPD experiencing an episode of exacerbation and requiring mechanical ventilation were admitted to the ICU and other milder forms of patients with COPD were treated on the floor ward. After weaning from mechanical ventilation, patients will be transferred to the floor ward. Patients with COPD admitted to the ICU due to other reasons such as major surgery, ischaemic heart disease and renal failure will be excluded. Other exclusion criteria are: (1) moribund and expected to die within $48 \mathrm{~h}$; (2) patients or their next-of-kin sign the do-not-resuscitation order (3) patients on vasopressors.

\section{STUDY PROTOCOL AND SOME DEFINITIONS}

All patients included into our cohort are followed up. On ICU entry, laboratory measurements including blood gas, chemistry profile, blood count and $\mathrm{C}$ reactive protein are obtained and recorded. Arterial blood gas includes the $\mathrm{pH}$ value, partial pressure of oxygen and carbon dioxide, base excess and lactate. Oxygen supplementation $\left(\mathrm{FiO}_{2}\right)$ is recorded. Liver function is reflected with total bilirubin and renal function is reflected by serum creatinine. White cell count, percentage of neutrophils and $\mathrm{C}$ reactive protein are obtained to represent the severity of infection. Demographics such as sex, age on admission, Acute Physiology and Chronic Health Evaluation II (APACHE II), duration of COPD and ventilation are recorded. Cardiac dysfunction will be assessed by troponins and B-type natriuretic peptide.

ICU mortality is defined as death during ICU stay and hospital mortality is death during hospital stay. ICU length of stay is defined as the time interval between ICU admission and ICU discharge. Severe critical illness may cause death early in the course of the disease, resulting in a short ICU stay; however, these patients do not present a favourable outcome. Thus, ICU length of stay will be measured and assessed as a clinical outcome only in survivors. For patients discharged from the ICU but readmitted to the ICU within $48 \mathrm{~h}$, it is considered as one episode of ICU stay. Mechanical ventilation included invasive and non-invasive ventilation with a positive end expiratory pressure $>5 \mathrm{~cm} \mathrm{H}_{2} \mathrm{O}$. For patients with invasive mechanical ventilation, a $1 \mathrm{~h}$ spontaneous breathing trial (SBT) is performed on a daily basis for the purpose of weaning. ${ }^{15} 16$ An SBT is considered to have failed if (1) arterial oxygen saturation $<90 \%$, (2) respiratory rate $>35 / \mathrm{min}$, (3) heart rate $>40 / \mathrm{min}$, (4) sustained increase or decrease in systolic blood pressure $>200 \mathrm{~mm} \mathrm{Hg}$ or $<80 \mathrm{~mm} \mathrm{Hg}$, (5) there are signs of distress or diaphoresis. Diagnosis of weaning failure is performed by intensivists blinded to the TTE findings. Successful weaning is defined when a patient weans from MV and breathes spontaneously on his/her own for $>48 \mathrm{~h}$. Duration of mechanical ventilation is defined as the time from initiation of ventilation to successful weaning.

\section{ECHOCARDIOGRAPHY}

TTE is performed immediately (within $6 \mathrm{~h}$ ) after ICU admission by using a portable ultrasound machine (Sonosite, M-Turbo). Experienced intensivists with more than 5 years of echocardiographic performance will perform the ultrasound measurement. The patient assumes a decubitus position or left lateral recumbent position to facilitate image acquisition. Left ventricular ejection fraction (LVEF) is assessed using linear dimensions derived from a two-dimensional image in a parasternal long axis view. However, when there is a marked regional difference in function, LVEF is assessed by using Simpson's rule. ${ }^{17}$ Mitral valve E-point to septal separation (EPSS) is also measured to assess the left ventricular function, and this is done in the parasternal long axis view. ${ }^{18}{ }^{19} \mathrm{E}$ wave velocity and $\mathrm{E}$ wave deceleration time are assessed using the pulse-waved Doppler analysis of mitral inflow in an apical four-chamber view. A wave velocity is obtained with the same method. The myocardial velocity is obtained by using tissue Doppler imaging (TDI) by placing the sample volume at the junction of the left ventricular wall with the mitral annulus of the lateral myocardial segments from the apical four-chamber view. $\mathrm{E}^{\prime}$ and $\mathrm{A}^{\prime}$ are obtained in this way. Since the $\mathrm{E} / \mathrm{E}^{\prime}$ ratio has been found to have important prognostic value and is a good non-invasive surrogate of left ventricular diastolic 
pressure, ${ }^{20-22}$ it is calculated and will be incorporated into a multivariable model. The mean of three measurements will be used as the final value. If a patient has atrial fibrillation, the $\mathrm{E} / \mathrm{E}^{\prime}$ ratio is averaged over 10 cardiac cycles. ${ }^{23}$ Right ventricular systolic pressure (RVSP) will be measured if tricuspid regurgitation is present in the apical four-chamber view or the subcostal view. RVSP is estimated by using the modified Bernoulli equation. ${ }^{24}$ The right atrial pressure (RAP) is estimated by using the diameter of the inferior vena cava (IVCD): RAP is estimated to be $5 \mathrm{~cm} \mathrm{H}_{2} \mathrm{O}$ if IVCD $<12 \mathrm{~mm}$, and is estimated to be 10 if IVCD $\geq 12 \mathrm{~mm} .{ }^{25}$ Central venous pressure is used to represent RAP if a patient has the central venous catheter in place. Other indices of right ventricular performance including RV fractional area change, the ratio of RV to LV end-diastolic area and RV eccentricity index will be measured. Furthermore, functional indices of RV systolic function such as tricuspid annular plane excursion or systolic peak velocity on TDI may also be informative on the role of $\mathrm{RV}$ function in mechanically ventilated patients with AECOPD and will be included in our protocol. Since patients with COPD are usually old, hypertensive and heavy cigarette smokers, they may suffer from known or occult coronary artery disease resulting in myocardial ischaemia during AECOPD. Regional wall motion abnormalities (RWMAs) will be evaluated on TTE. ${ }^{26}$ Briefly, the left ventricle is divided into $17 \mathrm{seg}$ ments (6 basal, 6 mid-ventricular, 5 apical), and each segment is evaluated and scored by motion and systolic thickening as follows: 1=normal/hyperkinesis, 2=hypokinesis, $3=$ akinesis, $4=$ dyskinesis, $5=$ aneurysmal. LV wall motion score index (WMSI) is calculated from the sum of all scores divided by the number of segments. ${ }^{27} 28$

\section{FOLLOW-UP STUDY}

The first evaluation of cardiac function with ultrasound was performed under critically ill condition in which myocardial ischaemia and RWMAs are prevalent. Furthermore, in the clinical setting of severe AECOPD requiring mechanical ventilation, numerous parameters may induce or deteriorate the pre-existing pulmonary hypertension, including hypoxic vasoconstriction, mechanical stress of hyperinflated lungs, loss of capillaries and inflammation. Therefore, a follow-up echocardiography will be performed for patients undergoing a spontaneous breathing trial (SBT).

\section{STATISTICAL ANALYSIS}

The primary end point in our study is the ICU mortality, and the sample size calculation was based on this end point. Sample size calculation was performed based on the Cox proportional hazard regression model. ${ }^{29}$ Statistical significance is defined as two-sided $\alpha=0.05$ and statistical power is $1-\beta=0.8$. The ratio of hazards of patients with $\mathrm{E} / \mathrm{E}^{\prime}>15$ to those with $\mathrm{E} / \mathrm{E}^{\prime}<15$ is presumed to be $3{ }^{3031}$ According to the previous report, we assume the prevalence of $\mathrm{E} / \mathrm{E}^{\prime}>15$ in patients with COPD is 0.15 . The corresponding binary covariate (patients with $\mathrm{E} / \mathrm{E}^{\prime}$
$>15$ and those with $\mathrm{E} / \mathrm{E}^{\prime}<15$ ) follows a Bernoulli distribution with the probability of a subject being in $E / E^{\prime}>15$ group, $p$, equal to 0.15 . As a result, the $\mathrm{SD}$ of the covariate is $\sqrt{\mathrm{p}(1-\mathrm{p})}=0.36$. Furthermore, our previous experience suggested that ICU patients with pulmonary disease had a probability of mortality rate of $20 \% .{ }^{13}$ The estimated sample size is 251 with an estimated required number of failure events of 51 .

Continuous variables are expressed as mean \pm SD or median and IQR as appropriate. Data of normal distribution are compared using the $t$ test and skewed data are compared using the non-parametric method (Wilcoxon rank-sum test). Categorical variables are expressed as a number and percentage, and are compared by using $\chi^{2}$ test.

All variables will be incorporated initially into the multivariable Cox proportional HR model as the full model. The main effect model is built by using the stepwise forward selection and backward elimination technique. The significance level for selection is predefined to be 0.15 and that for elimination is 0.2. However, we will not let the model building be completely determined by the machine. From a clinical perspective, important prognostic variables are forced to remain in the model if they are excluded by the stepwise procedure. These variables include sex, age and APACHE \|. Thereafter, we proceed to determine the specification of the model (proportional hazard assumption). It is equivalent to test the non-zero slope in a generalised regression of the scaled Schoenfeld residuals on functions of time. ${ }^{32}$ The estat phtest syntax in Stata will perform the test for each predictor as well as for overall predictors. If the test indicates violations to the proportional assumption, we sort to employ the Logistic regression model in which the end point is defined as the ICU mortality. The main effect model is built in the way as described previously. The relationship between $\mathrm{E} / \mathrm{E}^{\prime}$ and mortality in logit is examined by using the LOWESS smooth. If linear assumption is not satisfied, we will use the linear spline function to explore the relationship between $\mathrm{E} / \mathrm{E}^{\prime}$ and mortality. ${ }^{33}$ The knots for the linear spline function are chosen by examining the turning points in the lowess smooth curve. Another method is to transform the $\mathrm{E} / \mathrm{E}^{\prime}$ ratio into dummy variables, and the normal range of the $\mathrm{E} / \mathrm{E}^{\prime}$ ratio is used as the reference. ORs of different groups are reported against the reference. All possible interaction terms are examined for their statistical significance.

All statistical analyses will be performed by using Stata V.12.0 (College Station, Texas 77845, USA). Two-sided $\mathrm{p}=0.05$ is considered to be statistically significant.

\section{DISCUSSION}

Patients with COPD are at increased risk of developing cardiac dysfunction. It is reported that more than $20 \%$ of patients with COPD have concomitant cardiac dysfunction. ${ }^{34}$ Furthermore, observational studies have demonstrated that COPD is a strong risk factor for heart 
failure with a risk ratio of 4.5, and the association remains unchanged even after adjusting for other confounding factors. ${ }^{35}$ Although the underlying mechanisms for cardiac dysfunction in patients with COPD are largely unknown, the crosstalk is believed to be associated with over-activated inflammatory response, oxidative stress, pulmonary hypertension and airflow limitation. ${ }^{36-38}$ However, most studies are conducted in the general ward in which patients are less critically ill. The clinical implication of cardiac dysfunction in critically ill patients with COPD requiring tracheal intubation and mechanical ventilation is still largely unknown. The aim of the present study is to explore the prognostic value of cardiac dysfunction in patients with COPD requiring mechanical ventilation. The study setting is a mixed ICU and cardiac function is determined by using echocardiography. The primary study end point is the ICU mortality; and the secondary end points include hospital mortality, duration of mechanical ventilation, ICU length of stay and hospital length of stay.

Cardiac function assessed by echocardiography is multidimensional in that it includes systolic function, diastolic function and filling pressure. Systolic function is represented by EPSS and ejection fraction; diastolic function is represented by the $\mathrm{E} / \mathrm{A}$ ratio, $\mathrm{E}^{\prime} / \mathrm{A}^{\prime}$ ratio with TDI. Left ventricular filling pressure represented by the $\mathrm{E} / \mathrm{E}^{\prime}$ ratio has been extensively investigated for its association with clinical outcomes in a variety of diseases and settings such as myocardial infarction and patients with sinus rhythm. ${ }^{39-41}$ Thus, we aim to investigate the association between the $\mathrm{E} / \mathrm{E}^{\prime}$ ratio and clinical outcome in patients with COPD requiring mechanical ventilation.

AECOPD-induced cardiac disorders may be induced by myocardial ischaemia resulting from the oxygen supply-demand mismatch (due to hypoxia, tachycardia and fever). ${ }^{42} 43$ Our study will provide more insights into this mechanism by measuring the RWMAs. Pulmonary hypertension has long been known as a risk factor for poor clinical outcome in patients with COPD. ${ }^{44}$ It is evident that many patients with COPD have persistent pulmonary hypertension, and this can be exacerbated by hypoxic vasoconstriction, mechanical stress of hyperinflated lungs, loss of capillaries and inflammation during the acute care setting. In the present study, we will perform ultrasonographic studies in the acute and stable phases, aiming to separate the reversible and irreversible components of pulmonary hypertension.

The strength of the present study is the sound statistical analysis applied to model building. It is well recognised that the major limitation of an observational study is the influence of confounding factors. In our case, the prognostic value of $\mathrm{E} / \mathrm{E}^{\prime}$ may be affected by the variables age and APACHE $\|$, both of which are well established prognostic factors. The confounding effect can be adjusted by properly using the regression model. Furthermore, an important but frequently ignored issue in assessing the fitted model is the linear assumption. For many biological variables, a certain range, also known as the normal reference, may be associated with the lowest risk of poor outcome. Lower and higher values than this normal reference are associated with the increased risk of adverse outcome. Therefore, we will use the linear spline function to account for the nonlinear relationship.

If a certain aspect of cardiac function is demonstrated to be significantly associated with mortality, the clinical implication is that echocardiography can be routinely obtained for patients with COPD after admission to the ICU to better inform the clinical outcomes. Furthermore, interventions including fluid restriction, use of inotropes and diuretics may confer a beneficial effect when guided by echocardiography.

The limitations of the study should be acknowledged in several aspects. First, the study is observational in design and suffers inherent limitations of this kind such as selection bias and confounding effect. Second, echocardiography is problematic in mechanically ventilated patients with COPD in terms of quality, reproducibility and assessment of right ventricular function. Problems related to image acquisition may be solved by using transoesophageal echocardiography, but it is not available in the ICU where the study was conducted. Third, since multiple associations are to be tested in the study, it is subject to the problem of multiple testing, which may lead to false positive findings. Therefore, the result of the study is hypothesis generating and requires further confirmation.

Contributors ZZ conceived the idea and drafted the manuscript; LC conducted echocardiographic performance and interpreted the result (association between echocardiographic findings and clinical outcomes); KC performed statistical analysis and helped revise the manuscript; HN drafted the protocol for standard performance of echocardiography and was responsible for data acquisition. All authors approved the final version of the manuscript and were responsible for the integrity of the work. The study was funded by the Science and Technology Grants of Jinhua City (2013-3-008).

Funding This study is funded by science and technology grants of Jinhua City (2013-3-008).

Competing interests None.

Patient consent Obtained.

Ethics approval Ethics Committee of Jinhua Municipal Central Hospital.

Provenance and peer review Not commissioned; externally peer reviewed.

Data sharing statement This is a study protocol, so there are no data to be shared. All original data can be shared after the completion of the study. We will upload our data to the Dryad. At the present stage, there are no additional data available.

Open Access This is an Open Access article distributed in accordance with the Creative Commons Attribution Non Commercial (CC BY-NC 4.0) license, which permits others to distribute, remix, adapt, build upon this work noncommercially, and license their derivative works on different terms, provided the original work is properly cited and the use is non-commercial. See: http:// creativecommons.org/licenses/by-nc/4.0/

\section{REFERENCES}

1. Menezes AM, Perez-Padilla R, Jardim JR, et al. Chronic obstructive pulmonary disease in five Latin American cities (the PLATINO study): a prevalence study. Lancet 2005;366:1875-81.

2. Menezes AM, Perez-Padilla R, Hallal PC, et al. Worldwide burden of COPD in high- and low-income countries. Part II. Burden of chronic 
obstructive lung disease in Latin America: the PLATINO study. Int $J$ Tuberc Lung Dis 2008;12:709-12.

3. Lopez AD, Mathers CD. Measuring the global burden of disease and epidemiological transitions: 2002-2030. Ann Trop Med Parasitol 2006;100:481-99.

4. Mathers CD, Loncar D. Projections of global mortality and burden of disease from 2002 to 2030. PLoS Med 2006:3:e442.

5. Singanayagam A, Schembri S, Chalmers JD. Predictors of mortality in hospitalized adults with acute exacerbation of chronic obstructive pulmonary disease. Ann Am Thorac Soc 2013;10:81-9.

6. Grasso S, Leone A, De Michele M, et al. Use of N-terminal pro-brain natriuretic peptide to detect acute cardiac dysfunction during weaning failure in difficult-to-wean patients with chronic obstructive pulmonary disease. Crit Care Med 2007;35:96-105.

7. Mekontso-Dessap A, de Prost N, Girou E, et al. B-type natriuretic peptide and weaning from mechanical ventilation. Intensive Care Med 2006;32:1529-36.

8. Ma G, Liao W, Qiu J, et al. N-terminal prohormone B-type natriuretic peptide and weaning outcome in postoperative patients with pulmonary complications. J Int Med Res 2013;41:1612-21.

9. Zapata L, Vera P, Roglan A, et al. B-type natriuretic peptides for prediction and diagnosis of weaning failure from cardiac origin. Intensive Care Med 2011;37:477-85.

10. Lara TM, Hajjar LA, de Almeida JP, et al. High levels of B-type natriuretic peptide predict weaning failure from mechanical ventilation in adult patients after cardiac surgery. Clinics (Sao Paulo) 2013;68:33-8.

11. Moschietto S, Doyen D, Grech L, et al. Transthoracic echocardiography with doppler tissue imaging predicts weaning failure from mechanical ventilation: evolution of the left ventricle relaxation rate during a spontaneous breathing trial is the key factor in weaning outcome. Crit Care 2012;16:R81.

12. Papanikolaou J, Makris D, Saranteas T, et al. New insights into weaning from mechanical ventilation: left ventricular diastolic dysfunction is a key player. Intensive Care Med 2011;37:1976-85.

13. Zhang $\mathrm{Z}, \mathrm{Xu} \mathrm{X}, \mathrm{Ni} \mathrm{H}$, et al. Red cell distribution width is associated with hospital mortality in unselected critically ill patients. J Thorac Dis 2013;5:730-6.

14. Hurst JR, Wedzicha JA. Chronic obstructive pulmonary disease: the clinical management of an acute exacerbation. Postgrad Med J 2004;80:497-505.

15. MacIntyre NR, Cook DJ, Ely EW Jr, et al. Evidence-based guidelines for weaning and discontinuing ventilatory support: a collective task force facilitated by the American College of Chest Physicians; the American Association for Respiratory Care; and the American College of Critical Care Medicine. Chest 2001;120(6 Suppl):375S-95S.

16. Esteban A, Alia I, Gordo F, et al. Extubation outcome after spontaneous breathing trials with T-tube or pressure support ventilation. The Spanish Lung Failure Collaborative Group. Am J Respir Crit Care Med 1997;156(2 Pt 1):459-65.

17. Lang RM, Bierig M, Devereux RB, et al. Recommendations for chamber quantification: a report from the American Society of Echocardiography's Guidelines and Standards Committee and the Chamber Quantification Writing Group, developed in conjunction with the European Association of Echocardiography, a branch of the European Society of Cardiology. $J$ Am Soc Echocardiogr 2005;18:1440-63.

18. Pollick C, Pittman M, Filly K, et al. Mitral and aortic valve orifice area in normal subjects and in patients with congestive cardiomyopathy: determination by two dimensional echocardiography. Am J Cardiol 1982;49:1191-6.

19. Weekes AJ, Reddy A, Lewis MR, et al. E-point septal separation compared to fractional shortening measurements of systolic function in emergency department patients: prospective randomized study. $J$ Ultrasound Med 2012:31:1891-7.

20. Arques S, Roux E, Luccioni R. Current clinical applications of spectral tissue Doppler echocardiography $\left(E / E^{\prime}\right.$ ratio) as a noninvasive surrogate for left ventricular diastolic pressures in the diagnosis of heart failure with preserved left ventricular systolic function. Cardiovasc Ultrasound 2007:5:16.

21. Kim MK, Kim B, Lee JY, et al. Tissue Doppler-derived E/e' ratio as a parameter for assessing diastolic heart failure and as a predictor of mortality in patients with chronic kidney disease. Korean J Intern Med 2013;28:35-44.

22. Sharp AS, Tapp RJ, Thom SA, et al. Tissue Doppler E/E' ratio is a powerful predictor of primary cardiac events in a hypertensive population: an ASCOT substudy. Eur Heart J 2010;31:747-52.

23. Nagueh SF, Kopelen HA, Quinones MA. Assessment of left ventricular filling pressures by Doppler in the presence of atrial fibrillation. Circulation 1996;94:2138-45.

24. Douglas PS, Khandheria B, Stainback RF, et al. ACCF/ASE/ACEP/ ASNC/SCAI/SCCT/SCMR 2007 appropriateness criteria for transthoracic and transesophageal echocardiography: a report of the American College of Cardiology Foundation Quality Strategic Directions Committee Appropriateness Criteria Working Group, American Society of Echocardiography, American College of Emergency Physicians, American Society of Nuclear Cardiology, Society for Cardiovascular Angiography and Interventions, Society of Cardiovascular Computed Tomography, and the Society for Cardiovascular Magnetic Resonance endorsed by the American College of Chest Physicians and the Society of Critical Care Medicine. J Am Soc Echocardiogr 2007;50:187-204.

25. Jue J, Chung W, Schiller NB. Does inferior vena cava size predict right atrial pressures in patients receiving mechanical ventilation? $J$ Am Soc Echocardiogr 1992;5:613-19.

26. Citro R, Rigo F, Ciampi Q, et al. Echocardiographic assessment of regional left ventricular wall motion abnormalities in patients with tako-tsubo cardiomyopathy: comparison with anterior myocardial infarction. Eur J Echocardiogr 2011;12:542-9.

27. Cerqueira MD, Weissman NJ, Dilsizian V, et al. Standardized myocardial segmentation and nomenclature for tomographic imaging of the heart. A statement for healthcare professionals from the Cardiac Imaging Committee of the Council on Clinical Cardiology of the American Heart Association. Circulation 2002;105:539-42.

28. Lang RM, Bierig M, Devereux RB, et al. Recommendations for chamber quantification: a report from the American Society of Echocardiography's Guidelines and Standards Committee and the Chamber Quantification Writing Group, developed in conjunction with the European Association of Echocardiography, a branch of the European Society of Cardiology. J Am Soc Echocardiogr 2005;18:1440-63.

29. Schoenfeld DA. Sample-size formula for the proportional-hazards regression model. Biometrics 1983;39:499-503.

30. Park SJ, Lee SC, Jang SY, et al. E/e' ratio is a strong prognostic predictor of mortality in patients with non-valvular atrial fibrillation with preserved left ventricular systolic function. Circ J 2011;75:2350-6.

31. Kruszewski K, Scott AE, Barclay JL, et al. Noninvasive assessment of left ventricular filling pressure after acute myocardial infarction: a prospective study of the relative prognostic utility of clinical assessment, echocardiography, and B-type natriuretic peptide. Am Heart J 2010;159:47-54.

32. Schoenfeld D. Partial residuals for the proportional hazards regression model. Biometrika 1982;69:239-41.

33. Wood GA, Jennings LS. On the use of spline functions for data smoothing. J Biomech 1979;12:477-9.

34. Lainscak M, Hodoscek LM, Dungen HD, et al. The burden of chronic obstructive pulmonary disease in patients hospitalized with heart failure. Wien Klin Wochenschr 2009;121:309-13.

35. Curkendall SM, DeLuise C, Jones JK, et al. Cardiovascular disease in patients with chronic obstructive pulmonary disease, saskatchewan Canada cardiovascular disease in COPD patients Ann Epidemiol 2006;16:63-70.

36. Boussuges $A$, Pinet $C$, Molenat $F$, et al. Left atrial and ventricular filling in chronic obstructive pulmonary disease. An echocardiographic and Doppler study. Am J Respir Crit Care Med 2000;162(2 Pt 1):670-5.

37. Sin DD, Man SF. Why are patients with chronic obstructive pulmonary disease at increased risk of cardiovascular diseases? The potential role of systemic inflammation in chronic obstructive pulmonary disease. Circulation 2003;107:1514-19.

38. Eickhoff $P$, Valipour $A$, Kiss $D$, et al. Determinants of systemic vascular function in patients with stable chronic obstructive pulmonary disease. Am J Respir Crit Care Med 2008:178:1211-18.

39. Hillis GS, Moller JE, Pellikka PA, et al. Noninvasive estimation of left ventricular filling pressure by $\mathrm{E} / \mathrm{e}^{\prime}$ is a powerful predictor of survival after acute myocardial infarction. J Am Coll Cardiol 2004;43:360-7.

40. Nagueh SF, Mikati I, Kopelen HA, et al. Doppler estimation of left ventricular filling pressure in sinus tachycardia. a new application of tissue doppler imaging. Circulation 1998;98:1644-50.

41. Holland DJ, Prasad SB, Marwick TH. Prognostic implications of left ventricular filling pressure with exercise. Circ Cardiovasc Imaging 2010;3:149-56.

42. Beleslin BD, Ostojic M, Stepanovic J, et al. Stress echocardiography in the detection of myocardial ischemia. Head- to-head comparison of exercise, dobutamine, and dipyridamole tests. Circulation 1994;90:1168-76.

43. De Boer RA, Pinto YM, Van Veldhuisen DJ. The imbalance between oxygen demand and supply as a potential mechanism in the pathophysiology of heart failure: the role of microvascular growth and abnormalities. Microcirculation 2003:10:113-26.

44. Celli BR, MacNee W, Agusti A, et al. Standards for the diagnosis and treatment of patients with COPD: a summary of the ATS/ERS position paper. Eur Respir J 2004;23:932-46. 